\title{
THE BENEFIT OF USING SMART MANUFACTURING WITH ANALYSIS OF DIGITAL PRODUCT DEVELOPMENT: A REVIEW
}

\author{
Mareta Ramadhanis ${ }^{1}$ \\ ${ }^{1}$ Mechanical Engineering, Sekayu Polytechnic, South Sumatera, Indonesia \\ ${ }^{2}$ Mechanical Engineering, Sriwijaya University, South Sumatera, Indonesia
}

\begin{abstract}
Application product development and manufacturing using computer-aided technologies and what has already been implemented successfully in some segments of large-scale industry and suppliers. This study aimed to established a general understanding of the development of digital product development and manufacturing. The method of this study is a review of smart manufacturability analysis for digital product development. This study described product development is supported by CAD CAM and CAE programs from design and development with Cloud-Based Design with Manufacturing (CBDM). The results from this journal review are to know the factors that influence the results of 3D printing and how to fix these problems. The problem is by using a cloud base; there are still problems when translating digital forms into physical forms in the form of STL. or obj when using FDM (Fused Deposition Modeling). can be improved by using 3D SLA printer technology because SLA (Stereolithography) can produce good print results and complicated designs
\end{abstract}

Keywords: CAD, CAM, CAE, Digital product development, Cloud-Based Design and Manufacturing (CBDM)

\section{INTRODUCTION}

Digital products are developed through serviceoriented network products for consumers where consumers can configure and utilize services from the products of CAD products and redistribute with CAE [1]

The beneficial use of the CAD and CAM platform for companies or users who use it for hobbies from design can produce a 3D product with good results. one of the methods used is by producing fused deposition modeling (FDM) printers with consumer spend of $\$ 173.3 \mathrm{M}$ each year [2]

In this paper, it is expected to produce designs that can be realized with flawless products to avoid losses for companies or users of 3D printers using cloud platform methods.

The discussion will focus only on the FDM function, which will be applied cloud-based.

\subsection{Digital Manufacturability Analysis}

Traditional manufacturing is using features that can analyze Specifically on the CAD features section.

However, to me make parts with a complex design for manufacturing need to be translated into CAD model [3]

The reference used is some complexities that need to be that controlled technically to get back into the realm of digital CAD models FDM can translate well with printer results. There are good enough for complicated shaped modeling [4].

Some authors use the right approach to help the manufacturing process from a design created. [2]

The use model octree composition is used for this CAD model work on parts there are difficult to produce using additive and subtractive technology [5] exploited The basis used in this method is a graph then developed in the form of a manufacturability index for parts based on the sliced STL file input geometry. et al. then built a

*Corresponding author's email: coireta6@ gmail.com 
map whose function is to print the results in the form of 3D geometry using techniques from mathematical morphology [2]. The next process determines the print resolution, later on, to determine manufacturing size from features such as thin walls, protrusions, and holes [4]. An example of a cloud-based 3D printing assistant proposed by Rosen et al. Now allows users to upload. The STL file can change the area to be thinner with small features if the area is difficult to highlight by the user. The system has an advantage because it has a manufacturing assistantibillity so that good quality printing results.

\subsection{Digital Error Model}

Using the. STL file format is the primary standard, which is the de-facto standard of 3D printing technology. This format is by the surface of the CAD model with a triangle. Simple geometry is needed in this program, to make it easier when the STL file is exported in a free form so that the error when the $3 \mathrm{D}$ printer is getting smaller. When geometric increase is needed, processing that aims to get a good design before printing. Common problems that occur here, such as missing aspects, overlapping parts, and elements that are not neat. The .STL file shows several potential issues, including missing aspects, degenerate aspects, overlapping aspects, and non-manifesting topological conditions [6]. The main requirements for free mesh must be fulfilled from previous mistakes providing more info regarding the general manufacturing capabilities of the design.

\subsection{Fused Deposition Modelling (FDM) Process Limitations}

The results of FDM sometimes fail to represent the form of CAM due to the nature of the process in FDM itself. When the initial location occurs, then to the next point, the same error occurred, forming settling at the point $(\mathrm{x}, \mathrm{y})$ stopped from then to z. This creates geometric defects. Show in Figure 1 Seam caused by stop-start error in 3D-Printing. All layered manufacturing processes require the digital model to be divided into slices before the part can be manufactured. These slices then form the basis of a material deposition plan for the part [5] Slices can contribute to several errors that occur when comparing the original CAD model to the printed file. One example termed the stair-stepping effect occurs when the discretized contours of the $2.5 \mathrm{D}$ layers are printed. This phenomenon can significantly reduce the surface quality of the design

\subsection{Geometry Requirement and Printer Capability Mismatch}

The final design produces a model after 3D printing. The previous stage must first understand the capabilities of the target machine. The overhanging face that occurs in the design can be independent if the angle between the feature and the base plate is below a certain threshold. This limit is around $45^{\circ}$ for ABS materials. However, different materials and machines will have different values. Dimension accuracy is also a problem with FDM technology. Usually, the free set selected in machine software which cannot always be produced
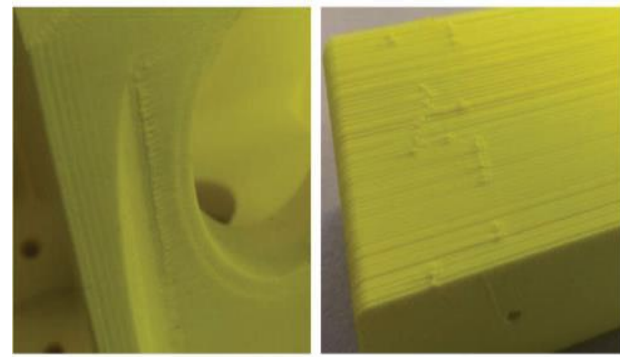

Figure 1 Seam caused by stop-start error in 3D-Printing.

Knowing the capabilities of the machine is the main stage that we prepare before using it.

\section{METHODOLOGY}

Methodology for this paper based on a review book and literature review. Cloud-based can be realized by assistant manufacturability, and it is first necessary to integrate cloud-based analysis into the CBDM platform model. There are two feedback options for these assistants: CAD and CAM-related feedback. CAD related feedback provides a system that can inform the designer of potential issues with their current design. The model can either be down to a problem with the STL mesh or the designers are aiming to manufacture features.

Cloud-based realized manufacturability assistant, it is first necessary to integrate cloudbased analysis into the CBDM platform model. There are two feedback options for these assistants: CAD and CAM-related feedback. CAD related feedback provides a system that can inform the designer of potential issues with their current design. This can either be down to a 
problem with the STL mesh or the designers are aiming to manufacture features that are outside the tolerances and capabilities of the machine.

Alternatively, CAM feedback would provide information regarding the suitability of the selected machine. For example, it could suggest selecting a printer that has a higher print resolution. A schematic of the cloud-based manufacturability tool methodology Figure 2

The software would have to understand the limitations of all the 3D printers that it has access to. By evaluating CAD geometry, it would be possible to highlight features that exist within the digital model that are outside the capabilities of 3D printer.

One method of realizing a smart manufacturability assistant is to integrate such a cloud-based analysis tool as a cloud-based middleware between the CAD and CAM systems. Analysis of the part would be achieved by analyzing specific AM features within the geometry. Any type of digital part decomposition should have the capability to identify specific areas of the design which fail to meet the machine capabilities and are therefore inherently non-manufacturable.

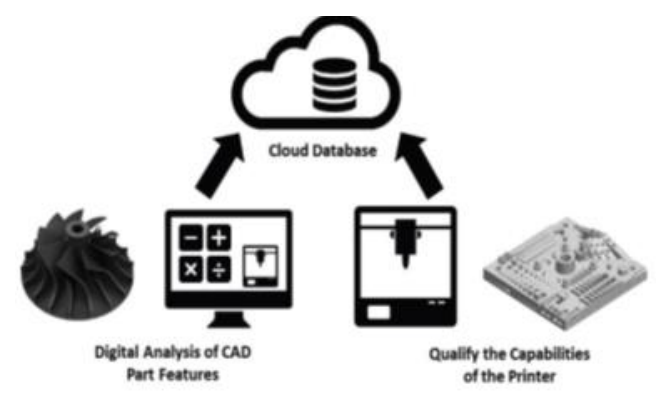

Figure 2 Example of feedback from a cloudbased analysis system into cloud-based $\mathrm{CAD} / \mathrm{CAM}$.

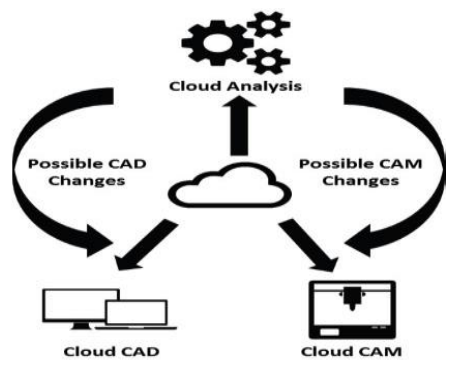

Figure 3 Schematic of a cloud-based manufacturability assistant features.
The result for the raw STL will be decomposed into STL files which then become slices that correspond to the thickness of the slices to be printed The maximum part dimensions can be calculated by computing the minimum bounding box volume of the STL file; these values can be used as an indication of whether the part will fit within the build volume of a selected printer.

Further information from the raw STL files such as facet normal can be used to calculate the angle between the digital part and the build

This equipment has the function to compile CAD files in the form of slices collected with the appropriate thickness so that it can detect thinwall geometries and fusible contour.

The proposed cloud-based manufacturability assistant, shown in Figure 3, works as a twostage process, existing in both the digital and physical worlds, unified by the cloud. |The first stage exists in the digital world; the CAD part is uploaded to the cloud-based analysis tool in the form of a. STL file. In the second stage, the printer capabilities are ascertained by printing and evaluating a physical test artifact. The first stage of the manufacturability tool may be realized as follows: An STL file is uploaded and checked to ensure that the mesh is free from errors described in section 2.2. The digital part representation can then be analyzed for its overall part dimensions and AM specific manufacturability features, including detection of thin regions and openings, excessive.

The user is then able to select an appropriate printer from the cloud that is believed to satisfy the requirements required to build the part. This can be an existing printer in the database which they have access to or a printer that they have quantified and added to the database. Alternatively, the user has full access to the database printer so they can use printouts for the desired print design. The printer is selected then the output of the analysis of manufacturability can be directly compared with the values obtained from the selected printer capability map. If the digital analysis shows that the part is within the tolerance determined from the printer capability map, then the part can be sent directly to the selected printer. The 3D printing assistant will realize by the method of interaction with the user is required. The method used must have intelligent characters that will provide feedback 
to users through a manufacturing analysis process and return feedback to users if needed.

\section{RESULTS AND DISCUSSIONS}

In this section, an overview of the work undertaken to fulfill the requirements for the second stage of the cloud-base manufacturability assistant, as defined in section 3, determines the capabilities of the 3D printer is discussed. A description of how the test part was designed and analyzed to develop the machine capability map will be described.

\subsection{Determining Part Capability Analysis Features}

A test part, as shown in Figure 4, was designed with 34 different feature sets. The part has a test upon the NIST standard test part with some additional features. These features include geometries, which would be of interest to the hobbyist and maker communities, including numbering and text. An overview of the feature requirements for producing the capability map is showing in Figure 5. This test part manufactured on a UP Box printer, which is representative of a mid-range desktop FDM machine. Three prints of the test part were produced and analyzed for each feature set. Feature sets are identified by numbers and are shown in Figure 4.

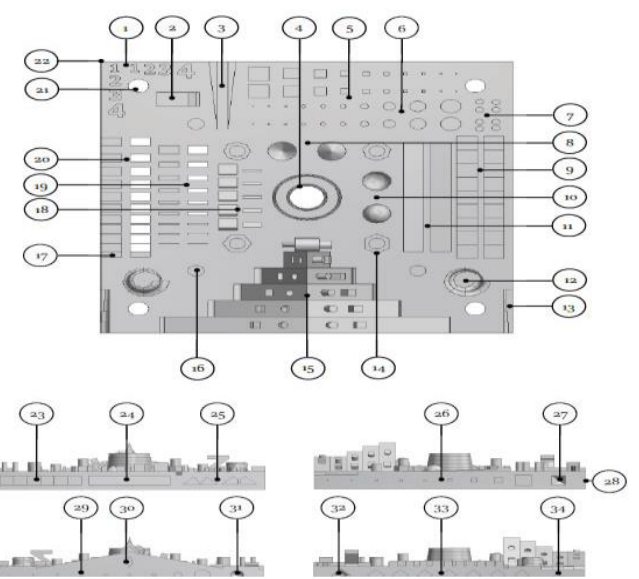

Figure 4 Machine Capability test part showing numbered analysis features

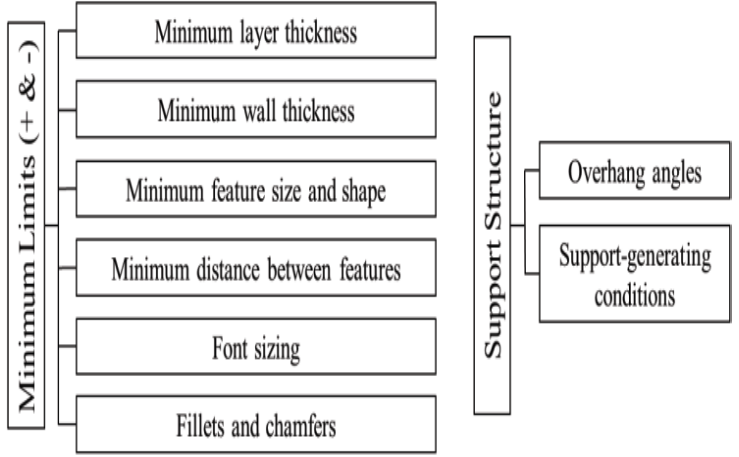

Figure 5 Test features required for producing a machine capability model

\subsection{Result from the Printed Test Part}

Printer capability is measured by qualitative and quantitative methods to meet the requirements of good printer quality. The tools used are Vernier caliper and micrometer for smaller ones using micrographs. Measurement with micrometer and Vernier caliper is very suitable, namely Leica M205. Show in Figure 5

\subsection{Capability Map for UP BOX Printer}

The results from the section were used to define the minimum and maximum capabilities of the printer for the measured features. Illustrates a capability map for a printer before it is uploaded into the cloud to form part of the cloud-based manufacturability assistant. This printability map includes details of material used, build dimensions of the printer, and the measured print geometry data.

\subsection{Limitations Of The Machines Capability Study}

The qualitative measurements within this study were performed using Vernier calipers, micrometers, and a microscope. As human interaction was required in taking the measurements, it is assumed that there could be a random error

Associated with the results. It is believed that low-cost methods of performing the quantitative analysis are preferable to using more accurate measurement techniques such as coordinate measurement machines or laser measurements. This is due to the requirement to populate the cloud-based manufacturability assistant with as many machine-material combinations as possible. It is believed that by keeping the barrier to entry in developing machines, capability reports low that many users will be able to add to the cloud-based analysis database. 


\section{CONCLUSIONS}

From this paper, we gain an understanding of how it works and the benefits of cloud-based and can be developed further for other tools.

There are some disadvantages when using a cloud base with FDM. This occurs when translated from the digital world. This includes problems with the STL format and an understanding of the physical capabilities of FDM printers. To overcome some challenges in creating a CAD that is suitable for manufacturing cloud-based manufacturing tools has been determined. Tool for comparing the minimum feature sizes contained in a CAD file and comparing them with the minimum size features that can be offered by a given $3 \mathrm{D}$ printer. If CAD files and printer capabilities are not compatible, feedback will be given to designers who can then improve the design or choose a different 3Dprinter model or process. Work is underway to develop a test section that can determine the capability maps for FDM printer material combinations. The results are displayed in a combination of relatively inexpensive, needed to fill the database with qualitative and quantitative information that will be appropriate to obtain the maximum capabilities of the 3D printer future. Besides that, you can try to use SLA printer technology that has advantages compared to FDM.

Work in this area will involve further development of cloud-based manufacturability assistant. One requirement will be to develop new algorithms that can gain an accurate assessment of the design features on an AM CAD model without using the STL file format and assess the user interaction with the tool.

The smart digital manufacturability assistant could be integrated into a cloud-based CAD system in which the user can request feedback on the manufacturability of the design during the design process. The work presented in this paper provides a new perspective on CBDM, where the cloud element is used as part of a knowledgebased appraisal method that will enable the user to gauge the printability of their part. This could have the effect of reducing the knowledge requirements necessary to ensure successful firsttime right $3 \mathrm{D}$ print builds.

\section{REFERENCES}

[1] D. Wu, D. W. Rosen, L. Wang, and D. Schaefer, "Cloud-Based Design and Manufacturing: A New Paradigm in Digital Manufacturing and Design Innovation," CAD Comput. Aided Des., vol. 59, pp. 1-14, 2015, doi: 10.1016/j.cad.2014.07.006.

[2] O. Kerbrat, P. Mognol, and J. Y. Hascoet, "Manufacturability Analysis to Combine Additive and Subtractive Processes," Rapid Prototyp. J., vol. 16, no. 1, pp. 63-72, 2010, doi: $10.1108 / 13552541011011721$.

[3] S. Goguelin, J. Colaco, V. Dhokia, and D. Schaefer, "Smart Manufacturability Analysis for Digital Product Development," Procedia CIRP, vol. 60, pp. 56-61, 2017, doi: 10.1016/j.procir.2017.02.026.

[4] G. A. O. Adam and D. Zimmer, "On Design for Additive Manufacturing: Evaluating Geometrical Limitations," Rapid Prototyp. J., vol. 21, no. 6, pp. 662-670, 2015, doi: 10.1108/RPJ-06-2013-0060.

[5] R. Ranjan, R. Samant, and S. Anand, "Integration of Design for Manufacturing Methods with Topology Optimization in Additive Manufacturing," J. Manuf. Sci. Eng. Trans. ASME, vol. 139, no. 6, pp. 114, 2017, doi: 10.1115/1.4035216.

[6] E. Asadollahi-Yazdi, J. Gardan, and P. Lafon, "Toward Integrated Design of Additive Manufacturing through a Process Development Model and Multi-Objective Optimization," Int. J. Adv. Manuf. Technol., vol. 96, no. 9-12, pp. 4145-4164, 2018, doi: 10.1007/s00170-018-1880-6. 